\title{
Acute generalized exanthematous pustulosis caused by an intravenous radiocontrast medium
}

\author{
Carolyn Michelle Tan MD, Jonathan Samuel Zipursky MD
}

n Cite as: CMAJ 2020 September 21;192:E1097. doi: 10.1503/cmaj.200364

A 58-year-old woman with a new diagnosis of acute myeloid leukemia underwent computed tomography (CT) of the chest and received $80 \mathrm{~mL}$ of intravenous radiocontrast medium (iohexol). Within 12 hours the patient had a body temperature of $40.1^{\circ} \mathrm{C}$ and exanthem characterized by diffuse erythema and edema on her face, trunk and limbs, with overlying pinhead-sized pustular lesions but no mucosal involvement (Figure 1). She had received contrast for a previous $\mathrm{CT}$ a decade earlier with no adverse reaction noted.

Laboratory investigations showed neutrophilia (12.7 [normal range 2.0-7.5] $\times 10^{9} / \mathrm{L}$ ), elevated creatinine level (192 [normal range 44-106] mmol/L) from a baseline ( $90 \mathrm{mmol} / \mathrm{L}$ ), no eosinophilia and negative results for blood cultures. We diagnosed acute generalized exanthematous pustulosis with a EuroSCAR study group score of 8 (see Table 2 in Sidoroff and colleagues ${ }^{1}$ ), consistent with definite acute generalized exanthematous pustulosis. ${ }^{1}$ Three days later, secondary purpuric changes and bullae developed on her legs (Appendix 1, available at www.cmaj.ca/ lookup/suppl/doi/10.1503/cmaj.200364/tab-related-content). Intravenous contrast was the most likely cause given the timing and absence of other exposures. Although our patient had received contrast previously, adverse reactions to contrast are unpredictable and can occur in patients who have previously tolerated radiocontrast media. Our patient was given topical steroids ( $1 \%$ hydrocortisone for her face and $0.1 \%$ betamethasone for her body), antihistamines and a 6-day course of prednisone, with resolution of the exanthem and acute kidney injury within 2 weeks.

Acute generalized exanthematous pustulosis is a severe cutaneous adverse reaction, and more than $90 \%$ of cases are attributable to drugs, most commonly penicillins and cephalosporins. ${ }^{2}$ Nondrug causes include infections (e.g., parvovirus B19 and cytomegalovirus), spider bites and herbal medications. Acute generalized exanthematous pustulosis from intravenous contrast is uncommon, with only 16 cases reported, of which 2 involved iohexol. ${ }^{3}$ Symptoms typically occur within 1-7 days of exposure. Classical findings include edematous erythema with overlying sterile, nonfollicular, pinhead-sized pustules, although purpura and bullae can also occur. ${ }^{1}$ Fever and neutrophilia are common, and mild acute renal failure has been reported in about one-third of patients. ${ }^{2}$ Once the causative agent is stopped, patients typically recover within 1-2 weeks without long-term sequelae. Topical steroids and antihistamines are used for symptomatic relief, with systemic steroids reserved for severe end-organ involvement. Referral for patch testing to confirm the allergy can be considered; however, false-negative results are possible, and the diagnostic value for severe cutaneous adverse reactions is unclear. ${ }^{4}$ Imaging modalities using iodinated contrast should be avoided given the reaction severity and risk of recurrence.

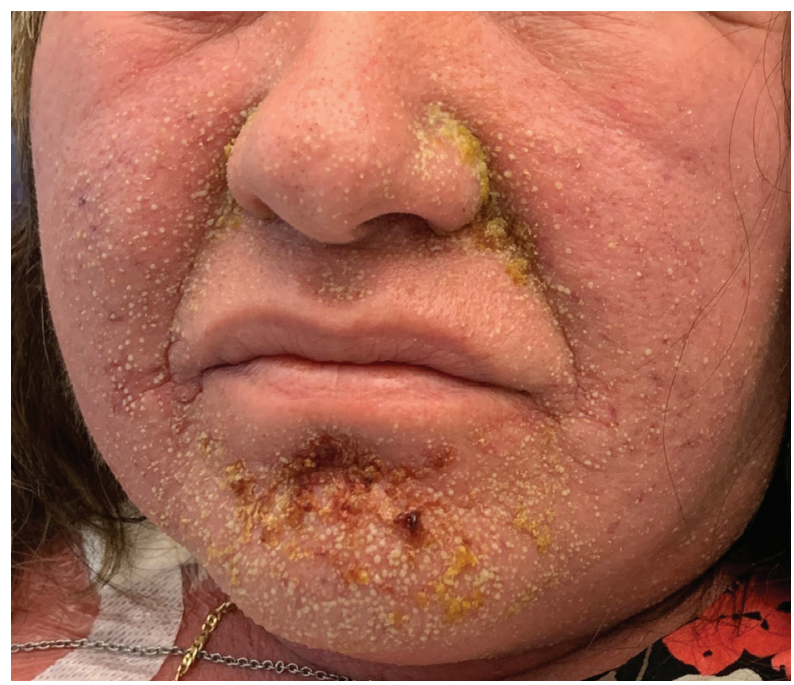

Figure 1: Edematous facial erythema with overlying nonfollicular, pinhead-sized pustules that occurred in a 58-year-old woman with acute myeloid leukemia who underwent computed tomography and was given intravenous iohexol.

\section{References}

1. Sidoroff A, Halevy S, Bavinck J, et al. Acute generalized exanthematous pustulosis (AGEP) - a clinical reaction pattern. J Cutan Pathol 2001;28: 113-9.

2. Leclair MA, Maynard B, St-Pierre C. Acute generalized exanthematous pustulosis with severe organ dysfunction. CMAJ 2009;181:393-6.

3. Velter C, Schissler C, Moulinas C, et al. Acute generalized exanthematous pustulosis caused by an iodinated contrast radiocontrast medium for computed tomography arthrography of the knee. Contact Dermatitis 2017;76:371-3.

4. Husain Z, Reddy B, Schwartz R. DRESS syndrome: part II. management and therapeutics. J Am Acad Dermatol 2013;68:709.e1-9.

Competing interests: None declared.

This article has been peer reviewed.

The authors have obtained patient consent.

Affiliations: Department of Medicine (Tan, Zipursky), Sunnybrook Health Sciences Centre; Department of Medicine (Tan, Zipursky); Division of Clinical Pharmacology and Toxicology (Zipursky); Institute of Health Policy, Management, and Evaluation (Zipursky), University of Toronto, Toronto, Ont.

Correspondence to: Carolyn Tan, carolyn.tan@sunnybrook.ca 